\title{
Factibilidad económica y financiera de un sistema de producción acuapónico de tilapia, lechuga y langostino de río en Delicias, Chihuahua, México
}

\author{
Economic and financial feasibility of an aquaponic production system \\ of tilapia, lettuce and river prawn in Delicias, Chihuahua, Mexico
}

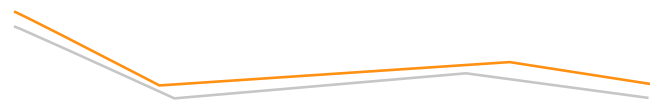

\author{
Dulce María Calderón-García*, Jesús Miguel Olivas-García*凶, \\ Concepción Luján-Álvarez*, Susana Hilda Ríos-Villagómez*, \\ Javier Hernández-Salas*
}

\begin{abstract}
Calderón-García, D. M., Olivas-García, J. M., Luján-Álvarez, C., Ríos-Villagómez, S. H., \& Hernández-Salas, J. (2019). Factibilidad económica y financiera de un sistema de producción acuapónico de tilapia, lechuga y langostino de río en Delicias, Chihuahua, México. Investigación y Ciencia de la Universidad Autónoma de Aguascalientes, 27(77), 5-11.
\end{abstract}

RESUMEN Con base en lo anterior, si sería factible producir las especies estudiadas en un sistema acuapónico.

La escasez de agua causa incertidumbre respecto al futuro desarrollo económico de la agricultura y ganadería, las superficies de siembra se han reducido significativamente y la demanda de alimentos es cada vez mayor. En tal sentido, la acuaponia se presenta como una alternativa, ya que es un sistema que combina la acuacultura y la hidroponia para la producción de alimentos, a la vez que se hace uso eficiente del agua dulce. El presente estudio evaluó la factibilidad de producir lechuga romana (Lactuca sativa L. var. Longifolia) en un sistema acuapónico, asociado al cultivo semiintensivo de tilapia (Oreochromis niloticus L.) y langostino de río (Macrobrachium rosenbergii D.) en Delicias, Chihuahua. Se concluye que se obtiene una ganancia promedio de 50 centavos por cada peso invertido a partir del segundo año de operaciones.

Palabras clave: acuaponia; hidroponia; rentabilidad; recirculación; alimentos; sustentable.

Key words: aquaponics; hydroponics; profitability; recirculation; food; sustainable.

\section{Recibido: 17 de julio de 2018, aceptado: 4 de marzo de 2019}

* Facultad de Ciencias Agrícolas y Forestales, Universidad Autónoma de Chihuahua. Km 2.5 Carretera Delicias-Rosales, Delicias, Chihuahua, México. Correo electrónico: kandi_calgar@hotmail.com; jolivas@uach. mx; clujan12@hotmail.com; srios@uach.mx; jhernans@uach.mx. ORCID: http://orcid.org/0000-0001-5906-6889; http://orcid.org/0000-0001-96575818; http://orcid.org/0000-0002-5869-766X; http://orcid.org/0000-00021085-7855; http://orcid.org/0000-0001-5846-915X

$凶$

Autor para correspondencia

\section{ABSTRAC1}

Water scarcity causes uncertainty for the future regarding the economic development of agriculture and livestock. Planting areas are reducing significantly and food demand is increasing. In this sense, aquaponics is an alternative, due it combines aquaculture and hydroponics for food production, while making efficient use of fresh water. The present study evaluated the feasibility of producing romaine lettuce (Lactuca sativa L. var. Longifolia) in an aquaponic system, associated to the semiintensive cultivation of tilapia (Oreochromis niloticus L.) and river prawn (Macrobrachium rosenbergii D.) in Delicias, Chihuahua. In conclusion, you get an average profit of 50 cents for each Mexican peso invested as of the second year of operations. Therefore, it would be feasible to produce the species in an aquaponic system.

INTRODUCCIÓN

La disminución en la disponibilidad de agua potable y el incremento en la necesidad de abastecimiento de alimentos son temas que cada vez adquieren mayor importancia. Un gran número de las unidades agrícolas productivas con riego no utilizan tecnología que optimice el uso del agua (FAO, 2000, 2014). Stover (2009) considera que la hidroponia, en 
issn 1665-4412, e-issn 2521-9758

Calderón-García, D. M., Olivas-García, J. M., Luján-Álvarez, C., Ríos-Villagómez,

S. H., \& Hernández-Salas, J. sistemas cerrados, y la acuaponia, cuyo propósito es la producción de alimentos, son innovaciones amigables con el medio ambiente. Además, indica que la acuaponia permite la limpieza del agua en el proceso productivo al aprovechar la excreta de los organismos acuáticos antes de la descomposición bacteriana, transformándola en nutrientes para el crecimiento de plantas y alimento para los langostinos de río.

La acuicultura, también llamada acuacultura, aporta $50 \%$ del pescado destinando a la alimentación en el mundo, con tasas anuales de crecimiento de $4.13 \%$ en los últimos años, por lo que contribuye a enfrentar el incremento de la población y del consumo de pescado per cápita, que en 2012 fue mayor a $19 \mathrm{~kg}$ anuales (FAO, 2018).

Rakocy (1999) señala que todo sistema acuapónico se enfoca en la producción, rentabilidad y el tratamiento de desechos, la considera una técnica novedosa y productiva, característica importante por la necesidad actual de conservar agua y a su vez producir alimentos nutritivos a un bajo costo y libres de químicos nocivos para la salud. Adicionalmente, la Universidad Pontificia Bolivariana (UPB, 2018) reporta que este sistema pudiera utilizarse para la producción simultánea de varias especies hortícolas como se muestra en la figura 1, con lo cual es posible producir diferentes alimentos animales y vegetales con la misma agua y nutrientes.

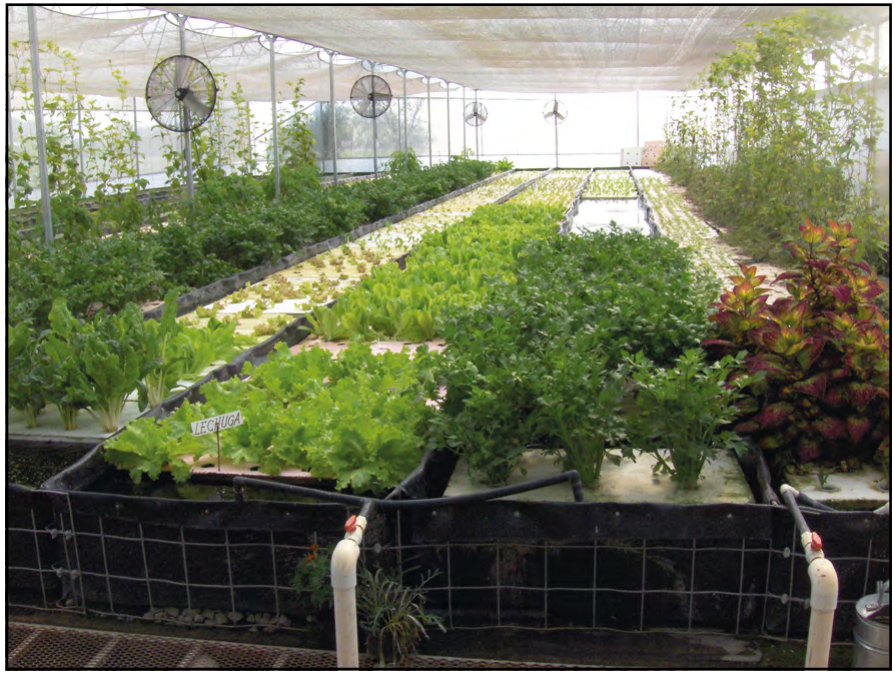

Figura 1. Policultivos en sistema acuapónico. Ejemplo de la producción simultanea de varias especies hortícolas en un mismo sistema acuapónico.

Fotografía tomada de UPB (2018).
El municipio de Delicias, Chihuahua se ubica en el Desierto Chihuahuense, tiene una precipitación media anual de $294.7 \mathrm{~mm}$ (INAFED, s. f.) y, aunque actualmente no existen referencias publicadas, se menciona que presenta un grave problema de salinización de los mantos acuíferos propiciado, entre otras razones, por la sobre explotación de pozos, lo cual trae como consecuencia la reducción de superficies de siembra.

El presente estudio evaluó la factibilidad de implementar el cultivo de lechuga romana (Lactuca sativa L. var. Longifolia) en un sistema acuapónico, asociado al cultivo de tilapia (Oreochromis niloticus L.) y langostino de río (Macrobrachium rosenbergii D.) en Delicias, Chihuahua, México.

MATERIALES Y MÉTIODOS

El estudio se realizó en el municipio de Delicias, Chihuahua, México; importante centro agrícola y punto estratégico con respecto a otros municipios de la zona por encontrarse en el centro del estado de Chihuahua y relativamente cercano a la frontera con EE. UU. Además, se considera que tiene potencial para la producción de alimentos frescos y de calidad tales como tilapia, lechuga y langostino de río, por contar con condiciones climáticas favorables para el desarrollo de dichas especies.

Se realizó un estudio de mercado, para lo cual se aplicaron 96 encuestas a jefas de familia con la finalidad de conocer la demanda potencial de los productos a elaborar y así determinar la capacidad requerida para el sistema productivo. El tamaño de la muestra se determinó con base en la fórmula para población finita de Murray y Larry (2009), con un error de muestreo de $10 \%$ y una significancia de $\alpha=0.05$. Se realizó un muestreo aleatorio simple para recopilar la información, la cual fue capturada y procesada en el software $R$ Studio versión 3.3.2. Igualmente, se determinó la infraestructura necesaria para el sistema acuapónico y se describió el funcionamiento de este, conforme a lo descrito por Caló (2011).

Por otra parte, conforme a la metodología de formulación y evaluación de proyectos de inversión (Baca Urbina, 2010), se detalló la tecnología necesaria para la construcción del sistema acuapónico, se determinaron los costos totales e infraestructura para la estimación de la inversión inicial, costos de 
producción, administración, venta y capital de trabajo. Para la evaluación económica y financiera se analizó la tasa interna de retorno (TIR), el valor actual neto (VAN) y la relación beneficio-costo (B/C), con lo cual, empleando los criterios del Grupo Banco Mundial (2017), se evaluó la viabilidad y factibilidad económica del proyecto.

Para identificar el comportamiento de los ingresos y egresos de las especies estudiadas (tilapia, lechuga y langostino de río), se elaboró un flujo de caja del proyecto en un horizonte de 10 años. Asimismo, para los indicadores financieros se utilizó una tasa de $10 \%$ en el análisis de sensibilidad bajo tres posibles situaciones, afectando ventas y costos de operación. Se aplicó igualmente un aumento en la tasa de $20 \%$ para el cálculo del VAN, que incrementa ventas y costos en $5 \%$.

\section{RESULTADOS}

\section{Estudio de mercado}

Se identificó que $67 \%$ de las familias del municipio de Delicias consumen filete de tilapia, 52\% lechuga, y solo $46 \%$ conoce el langostino de río; sin embargo, $53 \%$ de las jefas de familia manifiestan la posibilidad de consumirlo en sus hogares una vez que lo conozcan. En la tabla 1 se muestran los posibles factores que impactan en la demanda de cada uno de los productos estudiados.

De acuerdo con los resultados obtenidos se requiere producir anualmente la cantidad de 18.8 † de tilapia, 46,233 cabezas de lechuga y 1,680 kg de langostino de río. En la tabla 2 se observan las cantidades necesarias para producir por ciclo productivo, con una duración de 3 a 3.5 meses cada uno, de acuerdo con lo reportado por Calderón García et al. (2018).

Infraestructura necesaria. Para lograr la anterior producción se requieren dos estanques de geo membrana y cuatro estructuras para camas hidropónicas, entre otros insumos (figura 2), lo cual implica una inversión total de \$1,582,572.00.

El sistema NFT (Nutrient Film Technique) se conforma por dos contenedores (piletas) con capacidad de 18,600 I de agua y una cama nutritiva con capacidad de 3,853 plántulas para la producción de hortaliza por ciclo productivo, implementado en un área de $45 \mathrm{~m}^{2}$, y se emplean bombas para la recirculación del agua en los contenedores. En el sistema acuapónico, el agua sale del tanque de peces donde se contienen los desechos metabólicos de las especies acuáticas. El

Tabla 1

Motivos de bajo consumo de tilapia, langostino de río y lechuga en Delicias, Chihuahua, México

\begin{tabular}{lccc}
\hline Motivo & Tilapia (\%) & Langostino de río (\%) & Lechuga (\%) \\
\hline No gusta & 18.75 & 17.78 & 36.96 \\
Poca frescura & 43.75 & 35.56 & 54.35 \\
Precio alto & 37.50 & 26.67 & 8.70 \\
Falta de información & No aplica & 48.08 & No aplica \\
Poca presencia en el mercado & No aplica & 51.92 & No aplica \\
\hline
\end{tabular}

Nota: Elaboración propia.

Tabla 2

Requerimientos de producción de tilapia, langostino de río y lechuga por ciclo en Delicias, Chihuahua, México

\begin{tabular}{lccc}
\hline Producto & $\begin{array}{c}\text { Consumos con mayor } \\
\text { demanda (\%) }\end{array}$ & Cantidad necesaria & $\begin{array}{c}\text { Producción por ciclo } \\
\text { (De } 3 \text { a } 3.5 \text { meses) }\end{array}$ \\
\hline Tilapia & 32.29 & 12,540 alevines & $6,270 \mathrm{~kg}$ \\
Lechuga & 25.00 & 7,705 plantas & 7,705 cabezas \\
Langostino de río & 41.86 & 1,425 alevines & $560 \mathrm{~kg}$ \\
\hline
\end{tabular}

Nota: Elaboración propia con datos de Calderón García et al. (2018). 
issn 1665-4412, e-issn 2521-9758

Calderón-García, D. M., Olivas-García, J. M., Luján-Álvarez, C., Ríos-Villagómez,

8

S. H., \& Hernández-Salas, J.

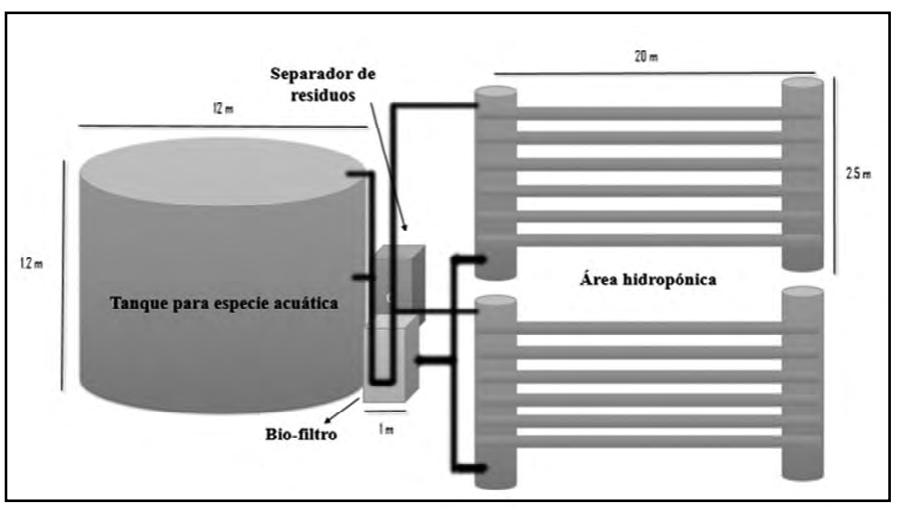

Figura 2. Diseño del sistema productivo bajo acuaponia. Nota: Elaboración propia.

agua primero pasa a través de un filtro mecánico que captura los residuos sólidos y posteriormente atraviesa un filtro que oxida el amoniaco a nitrato. Después, el agua viaja a través de la raíz de las plantas del cultivo (camas), donde estas absorben los nutrientes, y finalmente retorna agua purificada al tanque de peces, apoyada la circulación por bombas de agua.

Por otra parte, en cada tubo, con una separación de $25 \mathrm{~cm}$ entre cada plántula de lechuga, se mantienen 20 plantas. Así, la producción por ciclo productivo corresponde a 7,705 cabezas de lechuga, considerando que por cada ciclo de las especies acuáticas son dos de la especie hortícola. En el sistema es posible mantener a 12,540 alevines de tilapia anualmente, que se cosechan con un peso promedio de $500 \mathrm{~g}$ por ciclo productivo, con una duración de 3 a 3.5 meses por ciclo. Esto corresponde a 33 tilapias por $\mathrm{m}^{3}$. A la par se logra la engorda de 1,425 alevines de langostino anualmente, con un peso promedio de $390 \mathrm{~g}$ cada uno, de modo que se tienen 11 langostinos por $\mathrm{m}^{3}$. Bajo estas condiciones, para el análisis económico y financiero se consideró una tasa de mortandad de $3 \%$ en las tres especies. Los costos de los elementos requeridos para la implementación del sistema productivo se presentan en la tabla 3.

\section{Estudio económico y financiero}

En la tabla 4 se muestran los resultados de los indicadores financieros analizados de acuerdo con lo descrito previamente en el apartado referente a materiales y métodos.

Tabla 3

Inversión inicial para el sistema acuapónico

\begin{tabular}{lr}
\hline Concepto & Costo Total (M. N.) \\
\hline Diseño del sistema acuapónico & $\$ 45,000.00$ \\
Construcción del sistema & $\$ 20,000.00$ \\
Capacitación a trabajadores & $\$ 8,000.00$ \\
Renta de terreno (anual) (400 m²) & $\$ 287,070.00$ \\
$\begin{array}{l}\text { Requerimientos para producción } \\
\text { hortícola (lechuga) }\end{array}$ & $\$ 27,005.00$ \\
$\begin{array}{l}\text { Requerimientos para producción } \\
\text { acuícola (tilapia, langostino de río) }\end{array}$ & $\$ 129,036.00$ \\
Costos de producción & $\$ 629,569.00$ \\
Costos fijos & $\$ 327,240.00$ \\
Costos de energía eléctrica & $\$ 109,652.00$ \\
\hline TOTAL & $\$ 1,582,572.00$ \\
\hline
\end{tabular}

Nota: Elaboración propia.

Tabla 4

Cálculo de indicadores financieros en un sistema acuapónico

\begin{tabular}{lcccc}
\hline $\begin{array}{l}\text { Indicadores } \\
\text { financieros }\end{array}$ & $\begin{array}{c}\text { Aumento en CO' } \\
(15 \%)\end{array}$ & $\begin{array}{c}\text { Aumento en CO (10\%) } \\
\text { y Disminución en ventas } \\
(10 \%)\end{array}$ & $\begin{array}{c}\text { Disminución en } \\
\text { ventas (10\%) }\end{array}$ & $\begin{array}{c}\text { Aumento en tasa } \\
(20 \%)\end{array}$ \\
\hline $\operatorname{VAN}^{2}(\$)$ & 37,538 & 42,699 & 20,263 & 45,856 \\
$\operatorname{TIR}^{3}(\%)$ & 73 & 63 & 54 & 84 \\
Relación B/C & 1.36 & 1.46 & 1.22 & 1.98 \\
Flujo de caja (\%) & 2.37 & 2.69 & 1.28 & 2.98 \\
\hline
\end{tabular}

Nota: 1CO: Costos de Operación; 2VAN: Valor actual neto; 3TIR: Tasa interna de retorno; 4Relación Beneficio-Costo.

Elaboración propia. 
DISCUSIÓN

\section{Estudio de mercado}

La demanda potencial de las tres especies estudiadas muestra que existe un mercado atractivo para el sistema acuapónico propuesto, principalmente para la tilapia, de la cual, de acuerdo con CEC-ITAM (2006), la presentación en filete fresco es la más aceptada por el consumidor. Además, al ser la sexta especie en el consumo de pescado en EE. UU. (Arosamena-Villarreal, 2009), abre la posibilidad de aprovechar el mercado y obtener ventaja geográfica por la cercanía en la que se encuentra Delicias con respecto al mercado estadounidense, que de acuerdo con Josuepeit (2006) y FAO (2018), es el principal importador mundial, con aproximadamente 46,700 t anuales.

Por otra parte, el langostino de río se incluyó en el sistema para que actúe como limpiador al alimentarse de los residuos y excretas de la tilapia, lo cual lo hace atractivo para su producción y posible comercialización en la zona de estudio, ya que contribuye a hacer más eficiente el uso de la infraestructura y la conversión de alimento (Villarreal Colmenares \& Naranjo Páramo, 2008). De igual manera, Tidwell, Coyle y Bright (2010) reportan que estos sistemas contribuyen a que la tilapia mantenga bajas densidades de fitoplancton y de $\mathrm{pH}$ e incrementa la producción comparándola con la que se logra en monocultivos. Sin embargo, el langostino de río, rico en nutrientes, no se consume en Delicias por falta de información y ausencia en el mercado local.

Adicionalmente, la poca frescura de la lechuga ofertada en el mercado y el precio alto del filete de tilapia, impactan negativamente en la cantidad adquirida por las familias, como se muestra en la tabla 1. Existe una oportunidad de mercado en productos frescos y de alta calidad mediante el sistema acuapónico, lo cual coincide con lo reportado por Range \& Range (2005).

\section{Estudio económico y financiero}

El escenario optimista, con un aumento en ventas, bajos costos y una tasa de $20 \%$, proporciona un flujo de caja de $2.89 \%$ con respecto a la inversión y una ganancia de 98 centavos por cada peso invertido (tabla 4). Se garantiza la recuperación al presentar una TIR elevada. En el caso opuesto, al aumentar los costos de operación y obtener una disminución en las ventas de $10 \%$, es posible obtener una ganancia de 46 centavos, y un flujo de caja de $2.69 \%$, por lo que los sistemas acuapónicos son alternativas factibles al emplear la totalidad de los insumos requeridos en la construcción y procesamiento.

Con fines ilustrativos, se muestran en la tabla 5 las características detectadas que diferencian a tres sistemas productivos de la acuaponia, según entrevistas a productores del estado de Sinaloa,

Tabla 5

Comparativo de las características en tres sistemas productivos

\begin{tabular}{lccc}
\hline Concepto & Sistema Tilapia & Sistema Camarón & Sistema Acuapónico \\
\hline${\text { Costo de inversión inicial } \text { ha }^{-1}}_{\text {Presentan control de plagas y }}$ & $>\$ 200,000.00$ & $>\$ 250,000.00$ & $>\$ 150,000.00$ \\
enfermedades & No & No & Sí \\
Desperdicio de alimento & Sí & Sí & No \\
Desperdicios en estanques & Sí & Sí & No \\
Periodo de cosecha & $>3.5$ meses & $>4$ meses & 3 meses \\
Calidad de productos & Buena & Buena & Buena \\
Tiempo para observar & $<2$ años & $<2$ años & $<2$ años \\
ganancias & No & No & Sí \\
Amigable con el medio & Algunos & No & Sí \\
Ambiente controlado & Algunos & Algunos & Sí \\
Monitoreo & &
\end{tabular}

Nota: Elaboración propia. 
issn 1665-4412, e-issn 2521-9758

Calderón-García, D. M., Olivas-García, J. M., Luján-Álvarez, C., Ríos-Villagómez,

S. H., \& Hernández-Salas, J. por su importancia en la producción de camarón y tilapia.

Los resultados obtenidos muestran que la acuaponia es una alternativa factible y se coincide así con Rakocy (1999), quien indica que los sistemas acuapónicos son rentables y permiten tratar los desechos de manera eficiente. De igual manera Adler, Harper, Wade, Takeda y Summerfelt (2000), en un sistema de recirculación de trucha arcoíris y lechuga con albahaca dulce, obtuvieron una TIR de $12.5 \%$, mientras que en el presente estudio sistema acuapónico con tilapias, langostino de río y lechugas-, se obtuvo una tasa interna mayor a $50 \%$. Estos resultados demuestran la rentabilidad y eficiencia en la producción en masa, que además es amigable con el medio ambiente, coincidiendo así con Bogash (1997) y con Diver (2006).

Por otra parte, comparada la acuaponia con otros sistemas productivos, como acuacultura y agricultura, resalta lo siguiente:

- Los sistemas productivos cerrados presentan altos costos de inversión (Wheaton, 1982); sin embargo, la acuaponia, por hacer uso eficiente de los recursos, espacios y menor desperdicio de alimento, tiene la posibilidad de lograr la recuperación de la inversión a partir del segundo año de operaciones.

- Una de las características sobresalientes de la acuaponia, como mencionan Castilblanco e Hidalgo (2009), es la menor dependencia del clima, no existe efecto por variantes del suelo y es más eficiente el control de plagas y enfermedades, en comparación con la acuicultura y la agricultura tradicional.

- Comúnmente no es necesario el empleo de fertilizantes químicos e insecticidas durante el desarrollo del cultivo (Bogash, 1997; Diver, 2006), lo cual permite ahorros en insumos y garantiza alimentos saludables con valor nutrimental. Sin embargo, es importante tomar en cuenta que si llegara a presentarse alguna enfermedad o plaga, estos podrían propagarse más fácilmente.

- El flujo del proceso acuapónico proporciona los elementos necesarios para que el agua y los nutrientes se encuentren en movimiento (Graber \& Junge, 2009) y, por tanto, disponibles para las especies cultivadas.

- Es posible producir alimentos en menor tiempo en comparación con un sistema acuícola, con base en el control y monitoreo en la alimentación que se proporciona a las especies acuáticas (Adler et al., 2000).

Con base en lo anterior, y de acuerdo con las características climáticas del municipio de Delicias, es posible indicar que es factible la producción de tilapia y langostino de río en tres ciclos al año y seis ciclos de lechuga, esto con duración de tres meses para las especies acuáticas y seis semanas después del trasplante para la hortaliza. Sin embargo, es recomendable seguir realizando estudios in situ antes de implementarlo a nivel comercial.

CONCLUSIONES

La acuaponia puede ser una alternativa viable para la producción de alimentos frescos y nutritivos como filete de tilapia, lechuga y langostino de río de manera sustentable en Delicias, Chihuahua, México.

Al ser sistemas controlados pueden desarrollarse en espacios muy variados, siempre y cuando se tenga acceso a agua y energía eléctrica, y sea posible hacer una inversión inicial en infraestructura acorde con las necesidades del sistema; además de que se facilita el control de plagas y enfermedades en caso de que sea necesario.

El langostino de río es poco conocido por parte de las jefas de familia de Delicias, Chihuahua, lo cual, mediante estrategias de mercado, podría representar una ventana de oportunidad para ser integrado al consumo local.

La recuperación de lo invertido es posible en el segundo año de operaciones, con una ganancia promedio de 50 centavos por cada peso invertido.

Es factible la relación beneficio/costo de un sistema acuapónico con lechugas, tilapias y langostinos en diversos escenarios financieros.

\section{Agradecimientos}

Los autores desean agradecer al Consejo Nacional de Ciencia y Tecnología (Conacyt) por la beca otorgada al autor principal de la presente investigación para cursar estudios de Posgrado, así como a los revisores anónimos que con sus valiosas observaciones y sugerencias contribuyeron a mejorar la calidad del presente. 
- Adler, P. R., Harper, J. K., Wade, E. M., Takeda, F. \& Summerfelt, S. T. (2000). Economic analysis of an aquaponic system for the integrated production of rainbow trout and plants. International Journal of Recirculating Aquaculture, 1(1), 15-34. Recuperado de https://ejournals.lib.vt.edu/ijra/article/view/1359/1854

- Arosamena-Villarreal, D. (2009). Desarrollo de estrategias para la producción y comercialización de productos con valor agregado a partir de tilapia y aprovechamiento de los subproductos resultantes de su procesamiento (104 pp.). México, D. F.: Comité Sistema Producto Tilapia de México, A. C.

- Baca Urbina, G. (2010). Evaluación de proyectos (6a. ed.). México: Editorial MCGraw-Hill.

- Bogash, S. (1997). The Freshwater Institute Natural Gas. Powered Aquaponic System-Design Manual. West Virginia, US: The Conservation Fund Freshwater Institute.

- Calderón García, D. M., Olivas García, J. M., Legarreta González, M. A., Carrillo Soltero, M. E., Anchondo Nájera, J. A., \& Lujan Álvarez, C. (2018). Funcionamiento y viabilidad económica de un módulo de producción acuapónico en Los Mochis, Sinaloa, México. Custos e @gronegócio online, 14(3), 131-146. Recuperado de http://www.custoseagronegocioonline.com. br/quarenta\%20e\%20sete.html

- Caló, P. (2011). Introducción a la acuaponia. Argentina: Ministerio de Agricultura, Ganadería y Pesca. Recuperado de http://chilorg.chil.me/download-doc/86262

- Castilblanco, E., \& Hidalgo, J. (2009). Efecto de dos tratamientos de agua en la producción de lechuga (Lactuca sativa) bajo dos sistemas hidropónicos en piscicultura. Proyecto Especial del programa de Ingeniero Agrónomo de la Carrera de Ciencia y Producción Agropecuaria. Honduras: universidad Zamorano. Recuperado de https://buzonvirtual.zamorano.edu/vufind/ Record/RepoBWP-420/Description\#tabnav

- Centro de Estudios de Competitividad-Instituto Tecnológico Autónomo de México. (2006). Programa Maestro Nacional Tilapia (354 pp.). México: Autor.

- Diver, S. (2006). Aquaponics-Integration of Hydroponics with Aquaculture. North Carolina, US: ATTRA-National Sustainable Agriculture Information Service.

- Graber, A., \& Junge, R. (2009). Aquaponic systems: Nutrient recycling from fish wastewater by vegetable production. Desalination, 256(1-3), 147-156.

- Grupo Banco Mundial. (2017). Manual de préstamos para prestatarios del Banco Mundial [Documento en pdf]. Washington: Autor. Recuperado de http:// siteresources.worldbank.org/BORROWERPORTAL/Resources/ DisbursementHandbookSpanish061 107.pdf

- Instituto Nacional para el Federalismo y el Desarrollo Municipal. (s. f.). Enciclopedia de los municipios y delegaciones de México [Ficha con datos electrónica]. Chihuahua, México: Autor.
Recuperado de http://siglo.inafed.gob.mx/enciclopedia/ EMM08chihuahua/municipios/08021a.html

- Josuepeit, H. (2006). Aquaculture production and markers. Rome, Italy: FAO-ONU.

- Murray, R., \& Larry, J. (2009). Estadística (4a ed.). México: McGraw-Hill.

- Organización de las Naciones Unidas para la Agricultura y la Alimentación. (2000). El riego en América Latina y el Caribe en Cifras. Informe sobre temas hídricos 20. Roma: Autor. Recuperado de https://agua.org.mx/wp-content/ uploads/2017/08/El-riego-en-america-latina-y-el-caribe-encifras.pdf

$\bullet$

(2014). Superficie equipada para el riego [Diagrama en pdf]. Recuperado de http://www.fao.org/nr/water/ aquastat/infographics/Irrigation_esp.pdf

$\bullet$ (2018). El estado mundial de la pesca y la acuicultura 2014 [Detalles infográficos]. Recuperado de http://www.fao. org/resources/infographics/infographics-details/es/c/232019/

- Rakocy, J. (1999). The status of aquaponics. Aquaculture Magazine, Parte 1.

- Range, P., \& Range, B. (2005). Aquaponics helps to feed students, staff at orphanage in Reynosa, Mexico. Aquaponics Journal, 39, 18-19.

- Stover, S. (2009). Acuaponia: Técnica de producción superintensiva. Hortalizas. Recuperado de http://www. hortalizas.com/miscelaneos/acuaponia-tecnica-deproduccion-superintensiva/

- Tidwell, J. H., Coyle, S. D., \& Bright, L. A. (2010). Polyculture of Nile Tilapia, Oreochromis niloticus, either confined in cages or unconfined in freshwater prawn, Macrobrachium rosenbergii, ponds. Journal of the World Aquaculture Society, 41(4), 616625. doi: $10.1111 / \mathrm{j} .1749-7345.2010 .00402 . x$

- Universidad Pontificia Bolivariana. (2018). Acuaponía básica. Muestra de cómo en el sistema puede hacerse policultivos, muchas cosas con la misma agua, mismos nutrientes y misma temperatura [Fotografía]. Colombia: Universidad Pontificia Bolivariana. Recuperada de https://www.upb.edu.co/es/ formacioncontinua/curso-acuaponia-basica-

- Villarreal Colmenares, H., \& Naranjo Páramo, J. (2008). Cultivo de langosta de agua dulce Cherax quadricarinatus (Redclaw). Una oportunidad para la diversificación de la industria acuícola. Baja California Sur, México: CIBNOR, S. C. Recuperado de https://www.cibnor.gob.mx/images/stories/ biohelis/pdfs/Cultivo-de-langosta-de-Agua-Dulce-Cheraxquadricarinatus-Redclaw.pdf

- Wheaton, F. W. (1982). Acuacultura: Diseño y construcción de sistemas. México: AGT Editor, S. A. 